\title{
Adriana Chemello
}

\section{Luigia Codemo: appunti per una biografia intellettuale}

Abstract

La narrativa di Luigia Codemo ha una filiazione diretta dal romanzo storico manzoniano, da cui mutua l'interesse per le "genti meccaniche e di piccolo affare», ma attinge anche al vasto repertorio delle forme brevi, degli exempla, dei racconti morali, delle "storie vere" che sembrano riprese dalle colonne della «Gazzetta Veneta» di Gozzi, delle goldoniane chiacchiere da campiello, senza trascurare le storie sentimentali e romantiche dei racconti d'appendice. La cifra della sua scrittura sta in questa eterogeneità di moduli narrativi: racconti, scene, romanzi, drammi, bozzetti, che all'indomani dell'Unità d'Italia si sforzano di sopperire al bisogno di integrazione culturale e di pacificazione del "cuore" del popolo italiano. Una letteratura per il popolo che ambiva a «fare gli Italiani», spronandoli al bene e indicando loro un manipolo di «piccole virtù» sociali e domestiche, attente ai doveri verso Dio, la patria e la famiglia. Veicolo primario del processo educativo a cui Codemo si vota facendola diventare la sua "missione" è il libro che racconta fatti veri (per es. le biografie) oppure quelli verisimili (le «scene domestiche»), proponendo modelli credibili come l'operaio inventore Beniamino Franklin. Suggestionata dall'interesse romantico per i «buoni e gentili costumi del popolo», Codemo si prefigge di «educare ed ingentilire la moltitudine». Un capitolo inedito della sua biografia è rappresentato dall'attività filantropica che le ha fatto intersecare un protagonismo femminile emergente nella Venezia degli ultimi decenni dell'Ottocento. Donne nelle quali l'eredità mazziniana si stempera in azione pedagogicoeducativa, in particolare con le iniziative di Rosa Piazza e Laura Goretti Veruda per la formazione di buone madri e l'istituzione di scuole professionali femminili. Partecipando alle iniziative filantropiche veneziane degli ultimi decenni dell'Ottocento, Luigia Codemo realizza una forma di maternità vicaria in grado di compensare, almeno sul piano simbolico, la sua maternità biologica tragicamente spezzata dalla prematura morte del figlio.

Uno dei pochi, forse l'unico, ritratto di Luigia Codemo è quello uscito dalla penna del veneziano Cesare Musatti e apparso nelle colonne dell'«Ateneo Veneto», a pochi giorni dalla morte della scrittrice trevigiana, avvenuta a Venezia il 3 agosto 1898:

Chi la scontrava in sul tramonto lungo la riva degli Schiavoni, suo passeggio prediletto, secca, segaligna, solitaria; i capelli rifugiati in parte entro curiosa reticella, in parte a l'ombra di certo tocco ancora più curioso, la succinta vesta in aspra lite col figurino, il parasole inforcato sotto il braccio; e la vedeva ora ferma al parapetto d'un di quei ponti col ceruleo sguardo assorto nella magica visione, ora a un tratto, quasi scattando per occulta molla, galoppare verso casa con la furia d'un coscritto dopo la ritirata, non poteva ristarsi dal conchiuderne per un gran tòmo. (MUSATTI 1898, 245-60).

The e-journal «altrelettere» is hosted at the URL: http://www.altrelettere.uzh.ch, in accordance with the Open Access Policy of the University of Zurich. Please cite this article as follows: Adriana CHEMELLO, Luigia Codemo: appunti per una biografia intellettuale, in «altrelettere», 21.3.2012, DOI: 10.5903/al_uzh-4. 
Un ritratto in piedi della scrittrice steso con tratto affettuoso e partecipato, ripreso in parte da Antonio Caccianiga nell' «Illustrazione Italiana» con il ricordo della «figura originale [...] d'indole vivace, fantastica, indipendente d'ogni schiavitù». Ne risulta un profilo di donna laboriosa (parlando del suo lavoro, in una lettera a De Gubernatis, si definisce «un'operaia di buona volontà»), generosa d'animo, tenace e riconoscente nell'amicizia, che tuttavia non ne occulta le asperità del carattere, la diffidenza senile nei confronti di una società letteraria veneziana con cui si era trovata, in più occorrenze, ai ferri corti, adattandosi $o$ btorto collo a viverne ai margini. Ma il profilo restituisce della scrittrice trevigiana, migrata con il matrimonio a Venezia, il carattere risoluto, energico, poco disposto ad accondiscendere, fiero e intransigente con sé e con gli altri. Nata da un matrimonio «tra diseguali», aveva ereditato dalla madre Cornelia Sale e dall'ava materna Fiorenza Vendramin Sale ${ }^{1}$ una discreta sicurezza oltre ad una buona predisposizione per la scrittura letteraria, in cui effondeva $\mathrm{i}$ «diversi ideali in arte, in lettere, in politica» associandovi quasi con ostentazione «l'ideale della carità» (ARGONDIZZA 1898, 3).

Accostarsi all'opera di Luigia Codemo vuol dire trovarsi di fronte lo spettro della sua longevità associata ad una frenesia di scrittura dalla giovinezza, trascorsa nella natia Treviso e sorvegliata nella sua prima espressione da entrambi i genitori, al tempo sofferto della vecchiaia. E il lettore (o la lettrice) riscontrano differenze notevoli per tipologia e per carattere in queste esuberanti produzioni che lasciano però intravedere una coerenza intrinseca, una necessità vincolata al proprio destino biografico, pur restando «artisticamente incompiute». Sue virtù principali sono sicuramente la tenacia e la determinazione associate ad una coriacea perseveranza negli obiettivi, a volte penalizzata per la testarda sovraesposizione della propria attività letteraria. La sua indole di donna risoluta e non addomesticabile è espressa nella telegrafica definizione di sé offerta in una corrispondenza inedita con Raffaello Barbiera: «nata in condizione agiata, non aspiro che all'onore di esercitare una professione». Con la postilla: «mi è più facile comporre un quadro grande, compiuto con intreccio e copia di figure di quello che (sic!) piccole scritture, frazioni di studi» (lettera inedita a Raffaello Barbiera, da Venezia, 8.12.1865; BCTv, Ms. 1326/39). Dell'infanzia e della prima giovinezza rimangono le testimonianze affidate alle sue Pagine famigliari. Tra i primi ricordi ci sono le lezioni materne, le letture serali ad alta voce, da cui una 
misteriosa dolcezza penetrava nell'animo della fanciulla destandole sensazioni di piacevole armonia. All'iniziazione alla lettura si associa il ricordo di un giovane allievo del padre, Antonio Berti, che a lei bambina raccontava le fiabe e le storie di Guerrin Meschino. L'amore per i libri, per la lettura considerata ben presto un balsamo benefico contro le ingiurie della vita, pone le sue robuste radici nel periodo dell'infanzia trevigiana, nell'orgoglioso ricordo della nonna Fiorenza. ${ }^{2}$

La produzione letteraria di Luigia Codemo è abbastanza vasta e soprattutto non è concentrata intorno ad un unico genere letterario, bensì spazia tra tipologie di scrittura non omogenee. La sua presenza sulla scena letteraria dura più di quarant'anni, con un discreto successo di pubblico, come indicano le edizioni e le ristampe dei suoi titoli, anche se territorialmente circoscritto all'area trevigiana e veneziana. Non esiste, a tutt'oggi, una recensio completa delle diverse edizioni a stampa dei suoi racconti, romanzi, drammi, memorie, profili biografici, bozzetti, «pennellate» e simili, anzi la bibliografia che la riguarda non è esente da inesattezze e lacune, che spesso si tramandano da un contributo all'altro. 3 Impossibile, pertanto, presentare in modo cronologicamente ordinato una così ampia attività narrativa e saggistica, la cui configurazione può essere quella di una rapsodia che assembla racconti, schizzi, «scene» di genere, disegni e «pennellate», pagine in forma di saggio, reportage, senza trascurare le biografie di personaggi 'illustri' delle provincie venete, le memorie «famigliari» dedicate alla ricostruzione accurata dell'albero genealogico e ai resoconti dei viaggi in Italia e in Europa della famiglia Codemo.

Le memorie d'un contadino. Scene domestiche, pubblicate in due tomi nel 1856, recano la dedica: «Al diletto consorte». E nel frontespizio, a mo' di epigrafe, e di esplicita dichiarazione d'intenti, una frase da Shakespeare, «... tutto è vero» (CODEMo 1856). Scandite in otto "periodi» che marcano altrettante epoche della vita del protagonista Menico, queste «scene domestiche», avverte l'autrice, non sono un romanzo ma più semplicemente «un giornale» dove la "pittura de' costumi» della gente dei campi cede presto alla «storia [...] delle varie passioni» dei personaggi e alla loro rappresentazione, fedele all'assunto manzoniano da lei parafrasato: «il cuore umano è un abisso di contraddizioni». La lunga narrazione è alternata da pause descrittive, sermoni moraleggianti, «parentesi», «di- 
gressioni», «citazioni storiche», veri e propri «giornali di viaggio» e scritture private intitolate «frammenti dell'anima». Una «biografia» immaginaria, esemplata sulla tradizione lavorista di Beniamino Franklin, nella quale il campagnolo Menico racconta in prima persona la sua emancipazione sociale, dal fortunoso incontro con un misterioso cavaliere che lo toglie dall'ambiente campestre, lo fa educare a Venezia prima e a Firenze poi. Una lunga storia dove agli scenari campestri d'esordio, rappresentazione realistica degli usi e costumi campagnoli del «povero villanello delle Alpi», subentrano intricate e un po' farraginose vicende di amore e morte, secondo il formulario e i moduli della letteratura sentimentale ottocentesca. Le Memorie vengono segnalate con elogi dal «Crepuscolo» di Tenca nel giugno 1856, e, in Francia, dalla «Revue des deux mondes».

I racconti successivi pubblicati nel biennio tra il 1858 e il 1860 (Codemo 1858 ; 1860a; 186ob) vanno a comporre un'interessante trilogia di «scene domestiche» evidenziata soprattutto dall'apparato paratestuale della seconda edizione (1874-1875) che presenta lo stesso identico frontespizio, in cui viene modificato solo il titolo, inserito in una cornice ovale contornata da una filza di «quadri» e di figurine minuscole. Sormonta la cornice un frontone che riporta nel timpano l'indicazione «scene di vita». L'accostamento di «scene» e «cronaca» lascia intendere l'assenza di una narrazione ad intreccio sostituita da sequenze di quadri giustapposti, quasi un resoconto stenografico di conversazioni «domestiche» in grado di produrre un effetto di realtà, come in un susseguirsi di scene teatrali. Il reticolo narrativo piega a tratti verso la dimensione diaristica o la scansione memoriale, lasciando intercalare la debole trama da digressioni moraleggianti, intrusioni autoriali o appelli al lettore; a dominare ricomponendo il disegno della storia è il dialogo, il discorso diretto che mima il parlato nelle interiezioni, nelle ridondanze, nelle ripetizioni, nel rispetto dell'epigrafe shakespiriana dell'esordio, mentre l'autrice riserva a sé lo spazio di «umile cronista a cui spetta una cosa sola: la verità» (CODEMO 1875, 397).

Dopo la prima trilogia, la scrittrice mette da parte la finzione dell'anonimo narratore ma trova congeniale muoversi nella direzione delle "storie vere" prese al diminutivo, le uniche in grado di consentirle un’incursione nella realtà sociale del Veneto di fine Ottocento. Codemo si improvvisa osservatrice e pittrice di «scene di vita», seguendo in una sorta di «ciclo», su imitazione di Balzac, le diverse condizioni e appartenenze sociali. L'osservazione del reale è resa in forme 
colorite e variegate (che risentono della pratica pittorica appresa da Rosa Bortolan), la scrittura è un modo di dipingere con le parole scene e situazioni, un piccolo caleidoscopio o un museo colorato capace di trattenere impressioni penetranti. Le vicende narrate in questi libri girano attorno ad un nucleo di gesti e sentimenti inquieti, a tratti con un eccesso di ferialità, capaci però di riprodurre storie ancorate ad una realtà provinciale campagnola e cittadina ma sempre domestica, agita e osservata nell'hortus conclusus del privato. Restia ad avventurarsi nel mare periglioso del romanzo e del romanzo storico in particolare (a cui presta tuttavia un occhio di riguardo), la narratrice trova proficuo non vincolarsi ad una struttura narrativa rigidamente codificata, preferendo concedersi una specie di erranza o "deriva" tra diversi moduli narrativi. Nei suoi racconti le storie si scorciano in «scene» (come recitano i frontespizi dalle Memorie d'un contadino del 1856 alle Scene marinare del 1879, fino alle Scene campestri, domestiche, storiche del 1886), che sopperiscono alla debolezza dell'intreccio con l'alternanza di situazioni e modalità di scrittura. E sullo sfondo, qualche cenno discreto, qualche allusione pudica alla storia delle guerre risorgimentali, alla tensione ideale che aveva sorretto la generazione protagonista della rivoluzione del 1848 .

Le prove narrative di Luigia Codemo si confrontano con l'onda lunga provocata dai movimenti che si contendono la scena degli anni trenta dell'Ottocento: la scuola manzoniana sostenitrice del romanzo storico e paladina di una letteratura con funzione pedagogica e morale; la scuola realista sviluppatasi oltralpe con Balzac, Hugo, Sand e Sue, dove la prospettiva storica propugnata da Manzoni vira decisamente verso la contemporaneità, per descrivere e rappresentare una realtà sociale del presente, con lo studio dei caratteri, delle passioni, dei costumi, e un'attenzione ai particolari anche i più feriali. La narrativa di Luigia Codemo è debitrice al romanzo storico manzoniano per scelte tematiche, ma attinge a moduli narrativi mutuati dal repertorio della tradizione letteraria italiana da Boccaccio a Silvio Pellico, dalle forme brevi della narrativa esemplare ai racconti morali di Soave, dalle "storie vere" che sembrano uscite dalle colonne della «Gazzetta Veneta» di Gozzi alle goldoniane chiacchiere da campiello, dalle biografie agiografiche alle storie sentimentali e romantiche, dalle appendici ai bozzetti. La cifra della sua scrittura sta in questa eterogeneità di moduli prodotto di spinte diversificate e, a tratti, contraddittorie. I suoi racconti, ambientati in 
massima parte nella contemporaneità, rievocano storie di un passato prossimo, una specie di memoriale aperto in cui sarebbe tuttavia inutile cercare, come invece riscontriamo nelle Confessioni di Nievo, quel continuo décalage dell'asse della memoria da privata a pubblica, oppure quel pathos amoroso che innerva il libro dello scrittore garibaldino.

Benedetto Croce, interrogandosi sull'utilità di studiare una «produzione inferiore che i posteri sogliono quasi totalmente obliare» (CROCE 1924, 238), notava che essa rimane pur sempre «un documento di storia» che consente, a chi sappia adeguatamente indagarlo, di «lumeggiare la storia della civiltà», vale a dire la storia delle idee, delle tendenze, delle preferenze e del modo di sentire e di leggere il mondo delle generazioni passate. Luigia Codemo con la sua produzione di racconti, scene, romanzi, drammi appartiene a quel continente 'minore' della letteratura popolare che all'indomani dell'Unità d'Italia fa dell'integrazione e della pacificazione del popolo italiano la propria bandiera. Una letteratura per il popolo che ambiva a «fare gli Italiani», indicando loro un manipolo di «piccole virtù» sociali e domestiche, una forma di disciplinamento sulle orme del «buon senso e buon cuore» alla Cesare Cantù, fedele ai doveri verso Dio, la patria e la famiglia. Veicolo primario del processo educativo a cui Codemo si vota facendola diventare la sua "missione" è il libro che racconta fatti veri (per es. attraverso le biografie) oppure quelli verisimili (le «scene domestiche»), proponendo modelli credibili come l'operaio inventore Beniamino Franklin. Suggestionata dall’interesse romantico per i «buoni e gentili costumi del popolo», nonché dal fervore culturale provocato dal manzoniano «libro per tutti», Codemo si prefigge di «educare ed ingentilire la moltitudine». Le è arrivata un'eco un po' confusa, attraverso il genitore e lo zio pedagogo ma anche dalla lettura diretta di Dall'Ongaro e di Tommaseo, delle discussioni sulla forma più idonea alla letteratura per il popolo. Senza entrare nel merito delle dispute ideologiche sull'«ufficio dello scrittore», che secondo Carlo Tenca dovrebbe essere interprete e «profeta» del nuovo pubblico costituito dalla classe «più numerosa e meno istruita», Codemo si accontenta di soddisfare l'istanza filantropica di far del bene e, animata da un sentimento forte di "carità" verso le classi inferiori, si dice convinta che «i libri son belli e buoni, ma le opere vive di carità e di abnegazione ne valgono cento in folio» (CODEMO 1872, 177). 
Assente in lei la tensione nieviana ad «accostarsi [scil.: al popolo] con reverenza non con isdegno ad insegnare altrui quel poco che la sa» (Nievo 1956, 60), ma anche l'empatia che attraversa le pagine di Caterina Percoto, di cui pure è profonda ammiratrice, Codemo si appaga nella sua sedentaria pratica di scrittura demarcando con rigore gli ambiti: «I contadini non somigliano a noi, gente romanzesca di città, gente nervosa, impressionabile, eccitabile, e sanno conservare nel discorso e nei gesti un aspetto di calma benché finta». Una voce un po’ fuori del coro nonostante i suoi sforzi per accordarsi e sintonizzarsi sulla lunghezza d'onda del sentire comune, ma proprio per questo una voce emblematica della difficoltà di mediare tra il conservatorismo e il moralismo cattolico e le istanze innovatrici della cultura liberale post-unitaria. Una voce tuttavia apprezzabile proprio per la sua singolarità.

In due lettere inedite indirizzate alla poetessa perugina Alinda Bonacci Brunamonti sul finire degli anni '70 (da Venezia, 30.7.1879; BCTv, Ms. 1326.1) Codemo paragona il suo lavoro a «una pittura aspra, reale a educare il popolo» e le sue corrispondenze a «un modo di conversazione paesano ed artistico insieme» (da Venezia, 27.2.1882; BCTv, Ms. 1326.1), mostrando un consapevole intento didascalico, orientato verso una dimensione pedagogico-popolare. Formatasi su un romanticismo minore intrecciato al tema patriottico, particolarmente presente nel Veneto degli anni '50 e '6o, Codemo si erge a «umile cantastorie» e a «fedel cronista» del vero mantenendosi aderente ad un realismo che individua in George Sand, Alessandro Manzoni e Caterina Percoto i modelli di riferimento. Lettrice di Balzac e grande ammiratrice di Giusti, di cui conserva con venerazione nel suo Album una breve dedica, è particolarmente attratta da coloro che hanno saputo raccogliere e preservare «il fiore dei precetti e della filosofia popolare» per far conoscere l'indole naturale e positiva del popolo e si propone di seguirne le tracce. Ciò che fa difetto nei suoi racconti è la capacità di far palpitare le vite contristate dei suoi personaggi come riesce magistralmente a fare Caterina Percoto.

Per entrare nelle scelte letterarie di Codemo è utile leggere il reportage del pellegrinaggio a Nohant, nei luoghi biografici e letterari di George Sand. Quelle sobrie «note di viaggio» assumono l'intonazione di quadri a «più soggetti» dove il collante è costituito dal numen loci attorno a cui, per accostamenti progressivi, prende forma una schietta pittura. Se la fama di George Sand è «universale», 
la sua gloria è «immortale», per questo la sua pur modesta «scritturetta» (come la definisce) aspira a farsi monumentum alla musa ispiratrice, dove nel basamento sono scolpite le massime sul modo di intendere la letteratura che deve «mettere in luce chi fa bene» (CODEMO 1884, 7).

In George Sand vede una figura simbolica con cui condivide alcuni tratti biografici, a partire dal matrimonio «tra diseguali» dei genitori, la scelta della campagna e dei suoi abitanti con il ciclo dei romanzi campestri, ma soprattutto la scelta di non confondersi con il naturalismo, assumendo il principio sandiano che «l'art n'est pas une étude de la réalité positive; c'est une recherche de la vérité idéale» (SAND 1846, 9). La scrittrice del Berry diventa per Codemo una leggenda incarnata. La letteratura è per entrambe la scelta di una professione e di una «vocazione», dove l'oblatività femminile si fa «opera di carità», estrinsecandosi nella responsabilità educativa e nella dedizione alle pratiche filantropiche. Codemo vede soprattutto realizzata in George Sand un'idea aristocratica di femminilità. La donna-artista rimane per lei l'eccezione e in quanto tale la affascina e la nobilita, facendone un simbolo, un'icona positiva. Spostandosi dalle brume primaverili di Nohant agli orizzonti solari riflessi nelle acque lagunari o nella provincia trevigiana, le atmosfere idilliche e romantiche dei racconti sandiani sfumano e si opacizzano in «scene» e quadretti dove torna a prevalere l'intonazione didascalica e moraleggiante.

La lezione manzoniana del vero storico trova una buona applicazione in Codemo nel racconto I miracoli del sentimento. Scene del primo sacco di Roma (Codemo 1871), ispirato dalla lettera di San Girolamo Ad Principiam, e incentrato sulla figura della nobile romana Marcella considerata l'iniziatrice del monachesimo femminile. Il racconto viene definito dalla scrittrice, in una lettera all'amica Giannina Milli (da Venezia, 5.5.1868, in PAGANO 1991, 578) «lavoro storico, non santo, ma cristiano; morale di certo e italiano nobilissimo», dove lamenta tra dispiaciuta e contristata la scarsa attenzione della società letteraria.

Probabile emulazione dei racconti di Tommaseo ambientati in tempi antichi, il racconto rappresenta una specie di apprendistato prima di affrontare il romanzo più impegnativo, quello in cui si fa «cronista» della rivoluzione del 1848 nel Veneto e a Treviso in particolare, pagando così il suo tributo di scrittrice alla cultura risorgimentale. La rivoluzione in casa. Scene di vita italiana, pubblicato a Venezia (CODEmo 1869), pone al centro i complicati scenari storico politici 
delle province venete nella fatidica primavera del quarantotto. Parafrasando la celebre pagina introduttiva ai Promessi sposi, Codemo scrive:

la storia si occupa degli avvenimenti in grande, registra le alte cause ed i risultati, non può tener conto della vita intima, delle trame scoperte, del parenchima doloroso, dove si andava sviluppando il prodotto della rivoluzione italiana.

La narratrice sceglie di raccontare la politica dalle «intime stanze» anziché «dal campo di battaglia, dalle mura cittadine, dalle barricate». Tutto arriva al lettore filtrato attraverso i dialoghi, vere «fughe di notizie» nella consuetudine del palcoscenico, che si svolgono su fondali scenografici «domestici»: dalla «politica in orto» del primo capitolo alla «politica in campo» (VIII), alla «politica in cucina» (XII), alla «guerra in casa» (XVI) dove la politica si rifrange negli spazi domestici, lacerando gli animi del cenacolo familiare con la contrapposizione ideologica tra padri e figli o tra fratelli e contaminando con i suoi «guasti orribili» la sacralità domestica. Dentro la cornice di un sentimentalismo patriottico esibito con baldanza, si muovono patrioti generosi e traditori, monarchici e repubblicani, tra fughe rocambolesche e improvvisi ritorni, tra fragori di battaglie di cui arrivano gli echi lontani ma anche qualche schioppettata. Questo «romanzo patriottico», molto amato dalla scrittrice, è l'unico a cui abbia lavorato per emendarlo e perfezionarlo, oltre la prima edizione. È il romanzo che la fa conoscere oltre i confini regionali, grazie anche al positivo epilogo della terza guerra d'indipendenza che annettendo definitivamente il Veneto al Regno d'Italia agevola il commercio e la circolazione libraria. Erminia Fuà Fusinato, ricevuto in dono il volume, ringrazia l'autrice esprimendole tutto il suo entusiasmo (lettera inedita, da Viareggio, 14.9.[1869], BCTv, Ms. 1326.1):

lo lessi da capo a fondo, e posso con tutta coscienza asserire ch'Ella narrò gli avvenimenti di quel tempo memorabile, con una maravigliosa evidenza, e con la più scrupolosa verità storica. Per cui ritengo che chi volesse dettare l'istoria del Veneto in quei momenti, nulla potrebbe fare di meglio che consultare $L a$ Rivoluzione in casa. Io riprovai, in grazia sua, le liete emozioni, e le dolorose trepidazioni d'una giovinezza che appunto quegli avvenimenti resero anticipatamente matura. Rivissi in quell'epoca memorabile che i nostri figli non potrebbero pienamente comprendere ove penne simili alla sua, non ne affidassero il verace ricordo a pagine imperiture. 4

Luigia Codemo non percepisce come sua vocazione quella di cambiare il mondo e neppure di abilitare le donne ad affermarsi come nuovi soggetti politici, seguendo la strada intrapresa con passione dalla sua "sorella in arte" Caterina 
Percoto. Pur attenta alle sofferenze delle «gente meccaniche» che animano le sue «scene di vita», Codemo non si preoccupa di interrogarsi sulle sperequazioni sociali. Non aspira a modificare le condizioni materiali di vita delle classi subalterne, bensì a proporle ad esempio di bontà, coraggio e rassegnazione. Il precetto evangelico del fare del bene che Codemo vede incarnato nel poverello di Assisi, è per lei l'unico caposaldo per un 'cristiano' progresso sociale. La democrazia nella nazione italiana, secondo la sua visione del mondo, deve essere «sana democrazia che significhi amore al popolo e non odio ai ricchi. Ma per conservare viva la fiamma dell'amore bisogna sacrificarsi con serio, continuato sacrificio. È unicamente ai forti che Iddio concede l'ineffabile dolcezza d'amare, ossia di vivere [...]. Sacrificio non egoismo» (CODEMO 1891, 25).

Il suo essere nel mondo sotto l'ombrello protettivo della precettistica cattolica si esprime in una pratica di scrittura capace di raccontare della vita non le brutalità o l'odore di clinica alla Zola, da lei considerati ostentazione di cinismo, bensì il dolore, lo «spasimo dell'anima», la vita occulta, le «agonie» generate dall'«alterarsi degli affetti umani». Nel suo orizzonte concettuale la famiglia è il «vero santuario» degli affetti e il cardine della società civile purché sia abitata da una donna capace di essere, oltre che generatrice, educatrice delle giovani generazioni. Questo spiega l'adozione del modulo narrativo delle «scene», il suo fil rouge per portare alla ribalta della cronaca tanti tipi diversi situandoli in contesti spazio-temporali diversificati: dalle scene domestiche alle scene di vita, dalle scene marinare alle scene di ferrovia.

A partire dagli anni '7o, Codemo si impone uno sguardo retrospettivo, una pausa riflessiva rispetto agli avvenimenti politici e risorgimentali che avevano avuto su di lei un forte impatto emotivo. Escono in questo lasso di tempo le pagine di memorie familiari e letterarie, una specie di «libro di famiglia» in cui recupera il filo delle sue radici, l'appartenenza ad una aristocrazia intellettuale, ad una genealogia illustre, condividendo con i lettori i percorsi intellettuali ed emotivi della sua Bildung, della vicenda umana e letteraria, quella a cui ha affidato il senso ultimo della sua esistenza.

Contemporaneamente si dedica a ricostruire una «storia letteraria moderna» delle province venete con il volume Fronde e fiori del Veneto letterario in questo secolo (CODEMO 1872). Un lavoro pretenzioso che mette a nudo le fragilità della sua formazione culturale, anche se ne parla in termini entusiastici, quasi 
esaltanti, in una lettera all'amica Giannina Milli (da Treviso, 5.3.1872, in PAGANO 1991, 577): «Che formidabile lavoro, mia buona - e cosa ci ho messo dentro di notizie, di critica, di racconti, di vita!... Per taluni ci sarà la provocazione, ma i buoni saran con me. E, se in ogni provincia d'Italia imiteranno il nostro esempio, si formerà un bel nucleo di storia letteraria moderna». Per fortuna l'esempio non ha avuto seguaci, ma la provocazione non ha tardato a far sentire le reazioni indignate come quella dell'erudito trevigiano Francesco Fapanni. È noto il duro giudizio da lei espresso nei confronti delle Confessioni nieviane. Stroncatura ingiusta e inopportuna, con in più il contraddittorio giudizio di valore sui due personaggi femminili di Clara e Pisana, considerati ottimi come «ritratti» ma «insopportabili quali eroine da romanzo» (CODEMO 1872, 142).

La sua firma è stata una presenza fissa, per circa un decennio, nella «Strenna Veneziana», una pubblicazione annuale stampata in un'elegante veste editoriale dalla veneziana Tipografia del commercio a partire dal 1862, per iniziativa di Eugenia Gentilomo Fortis e Giovannantonio Piucco. Le carte conservate nel suo archivio privato attestano gli sforzi compiuti per collocare le sue opere presso un editore di levatura nazionale. Nel 1866, per esempio, corteggia con insistenza l'editore Le Monnier di Firenze, sfruttando le entrature di Tommaseo. Ma il momento non è dei più favorevoli perché l'editore sta riconfigurando la struttura societaria della casa editrice, pertanto non può assumere nuovi impegni di stampa. Codemo non desiste, lascia passare un paio d'anni e poi, con la mediazione di Giannina Milli, propone, ancora una volta senza successo, la ristampa di suoi lavori all'editore fiorentino. Nonostante diversi tentativi per inserirsi in un circuito editoriale di più ampio respiro, le sue «scene di vita» e i suoi «quadri» fanno lavorare i torchi delle tipografie venete. Nel 1874 prova a sondare la disponibilità dell'editore fiorentino Barbèra ma il breve scambio epistolare non sortisce gli effetti sperati. L’invio dei suoi romanzi al direttore del «Corriere della sera», Eugenio Torelli Viollier, con la segreta speranza di una segnalazione sul giornale milanese, produce un cortese ringraziamento dal suo «antico lettore ed estimatore», ma non la agognata recensione. Scrive anche al Nigra, ambasciatore italiano a Parigi, sollecitandone l'interessamento per qualche segnalazione sulla «Revue des deux Mondes», ricevendo la risposta che la rivista è avara di segnalazioni anche con gli autori francesi (lettera inedita, BCTv, Misc. 
1326.1). Le corrispondenze private gelosamente conservate nel suo archivio testimoniano di una spasmodica ricerca di segnalazioni e recensioni in Italia e in Francia e di una altrettanto tenace autopromozione, con l'invio dei suoi lavori a riviste, biblioteche, scuole, provveditorati, accademie, municipi e quant'altro che in genere si limitano a ringraziare l'autrice per il cortese dono.

Ma Codemo non si lascia intimidire e continua a cercare una visibilità letteraria e a pubblicare i suoi libri con lo Zoppelli di Treviso. Libri che, soprattutto negli anni '8o sono frutto di un'operazione di collazione e di accumulo di moduli narrativi appartenenti a generi diversi. Ogni nuovo volume si configura come una specie di laboratorio alchemico in cui i diversi materiali lasciano intravedere le linee di una scrittura che usa soprattutto lo sguardo per registrare frammenti di realtà, spostando via via il proprio punto d'osservazione su scenari antropologici e naturalistici mobili.

Dopo la stagione dei lunghi racconti a «scene», Codemo sviluppa, quasi per contrappasso una narrativa della brevitas, del «pezzetto» alla Gozzi, «scene contemporanee» che intitola pennellate, per le quali può valere il giudizio da lei formulato per scritture simili di Pietro Chiari, capaci cioè di promuovere nel lettore «un innocentissimo buon umore, e chi non lo sa che il sorriso aggiunge un filo alla trama della vita?» (CODEMO 1875, 416). Sono «schizzi», piccole storie vere legate alla quotidianità, viste e osservate tra le calli e le fondamenta di Venezia, registrate con scrupolo documentario, mimandone il carattere pittoresco e vivace del fatto di cronaca. Senza indugi descrittivi, con qualche scorciato dialogo ad effetto per catturare la complicità con il lettore, le «pennellate», contratte in uno spazio minimo, sono efficaci "figurine" legate alla dimensione antropologica del presente. Una scrittura bozzettistica di debole tensione adatta per quella forma di utilizzo differenziato dalla rivista al libro. Una letteratura di minore estensione che si presta ad essere consumata dai lettori di giornali e periodici, certamente più numerosi di coloro che invece vincolano la lettura all'acquisto di un libro. Nelle intenzioni dell'autrice le pennellate vorrebbero essere piccoli «poemi» per far rivivere le tradizioni di vita marinaresca che per quattordici secoli hanno resa prestigiosa e prospera la Serenissima, «il centro d'un gran regno, sempre autonomo: e, ancorché atterrato, un colosso mostra negli immani frammenti delle sue forme cosa fu» (CODEmo 1872, 425). Tra que- 
sti frammenti e piccoli tocchi di pennello, Codemo auspica di riuscire a far rivivere l'«eredità gentilizia» delle conversazioni di Isabella Teotochi Albrizzi o dei Siti pittoreschi e prospettici delle lagune venete, usciti dalla penna di Giustina Renier Michiel. Accanto alla vita aristocratica dei tempi andati permangono le presenze forestiere all'Arsenale che conservano a Venezia lo statuto di città cosmopolita, e le vivaci attività fabbrili del popolo minuto e dei barcaioli che al ritmo cadenzato e vivace del remo modulano la voce sui versi di un Carrer o di un Fusinato. Senza mai abbandonare i recinti provinciali, o veneziani, Codemo per «l'amore alla mia Venezia» intraprende il quadro di genere e si fa reporter con il taccuino in mano, abbandona la dimensione domestica e, pur nello spazio ristretto della colonna di giornale, assapora l'esotico passeggiando lungo la riva degli Schiavoni, ammirando le corazzate in rada nel porto, facendo rivivere lo spirito della Venezia marinara dei secoli passati.

Ma suo primo reportage può considerarsi il racconto Dal mare alle Alpi, illustrazione d'un album, che viene pubblicato nella «Strenna Veneziana per l'anno 1870» (CODEMo 1870). Si configura come un lungo reportage di viaggio da Venezia a Fadalto, alla Valle d'Agordo ed è il resoconto di un viaggio compiuto con la famiglia. Lasciata alle spalle Treviso, la nostra viaggiatrice risale per un breve tratto il corso del Piave (Anasso) per spingersi fino a Conegliano utilizzando la strada ferrata; il viaggio prosegue poi in carrozza verso Vittorio Veneto. È il lembo nord orientale della provincia di Treviso che corre tra le Prealpi Trevigiane e una fascia collinare: una piccola unità geomorfologica dove la calma sinuosità delle colline prosegue nel massiccio slancio della montagna. Superato Vittorio Veneto e l'antico santuario di Santa Augusta di Serravalle (che verrà visitato sulla via del ritorno), le strade si fanno strette e disagevoli e s'inerpicano lungo valli laterali, fiancheggiando i laghetti del Meschio e poi il Lago Morto e quello di Santa Croce fino a Fadalto, e proseguendo nel Cadore fino a Belluno. Le suggestive descrizioni di scenari alpini, che incombono (spesso opprimendo l'animo del viaggiatore-pellegrino) come gli spalti di una fortezza nemica, cedono a tratti a scaglie narrative dove ritornano figurine familiari di popolani e montanari colti nei loro ripetitivi gesti quotidiani. Un universo osservato da un'angolazione un po' infantile, a tratti scolastica, ravvivato da un cromatismo tenue e leggero nei toni sia quando descrive la flora rupestre ed alpina sia quando registra i giochi di luci e di colori delle guglie dolomitiche riflesse negli specchi d'acqua dei 
laghetti montani, senza trascurare i manufatti umani, soprattutto quelli di pregio artistico. È il caso di Belluno con il Duomo, la chiesa della Madonna delle Grazie, il Campedel, il teatro e la villa Manzoni. L'altopiano del Cansiglio nelle Prealpi carniche, situato a cavallo tra le province di Belluno, Treviso e Pordenone, è al centro del reportage. Ad esso si accede da sud, appena superato Vittorio Veneto, e da nord nella zona dell'Alpago. Ma il viaggio prosegue lungo la Valle di Agordo segnata dal torrente Cordevole, affluente del Piave, fino al paese omonimo, e alle antiche miniere della Val Imperina da cui si estraeva il rame, dopo aver attraversato la Valle di Zoldo sovrastata dal Monte Pelmo, mentre all'orizzonte si intravedono le cime dell'Antelao e della Marmolada. Eugenia Pavia Gentilomo Fortis, in una lettera all'amica «Giggia» (diminutivo di Luigia), così si esprime dopo la lettura dell'Album (la lettera è data 28 febbraio 1880 e si conserva inedita tra le carte Codemo della Biblioteca Civica di Treviso):

Penna e pennello si scambiano spesso un fraterno saluto nella tua mano e non solo il saluto, ma tanto si voglion bene che scambiano anche fra loro i geniali uffici. Ora ho dinnanzi agli occhi la pittura a carta vergata d'uniformi caratteri d'inchiostro, delle Alpi Giulie. E godo la vista svariata di profondi abituri e delle audaci vette... Ecco l'uomo ecco il sentimento, ecco la morale del poema, cioè del quadro... Oh! La chiusa mi piace più di tutto; la chiusa, e per chiusa intendo parecchie strofe, compendi a nobilissimi affetti e pensieri, c'è il vero che mi piace più del verismo, lo sai perché io, nel mio nulla, come il bimbo anelo alla luce, alla pura luce del sole; e qual più bel sole del vero, che spande tra raggi sideri e pure idealmente belli?

Un'anticipazione delle «pennellate veneziane», che diventano la specializzazione della sua scrittura d'occasione e giornalistica, può considerarsi la plaquette stampata nel 1872 con il titolo Chioggia e Schio. Il reportage del viaggio a Schio, dopo un lungo preambolo sui compagni di viaggio in omnibus che considera «veicolo di confidenza», intrigante quanto un «isolato capitolo di romanzo», descrive la visita alla fabbrica alta dell'industriale scledense Alessandro Rossi, la «cattedrale» del lavoro, la «cara e preziosa Manchester d'Italia», e si chiude con un lusinghiero cenno al teatro Jacquard, fatto costruire da Rossi proprio a fianco della fabbrica, spazio dell'intrattenimento onesto ed educativo delle maestranze.

Le scritture estemporanee apparse tra la fine degli anni '8o e i primi anni '9o, da Un viaggio a bordo (1886) a I vaporetti (1891) fino alle Pennellate marinare del 1895 , in incerto equilibrio tra descrizioni e reportages, sono «vere scene 
contemporanee», siano esse campestri o domestiche, intime o artistiche. I temi veneziani riproposti in occasione dei suoi frequenti ritorni in laguna, negli ultimi anni della sua vita, si concentrano in «pennellate di verità e di fantasia» capaci di catturare piccoli particolari, architetture sporgenti dalle acque, piccole calli o antri bui, i gondolieri con le loro imbarcazioni che resistono spavalde all'arrivo dei vaporetti. La padronanza della tecnica dell'acquerello si percepisce nei modi in cui la città lagunare diventa oggetto di rappresentazione. Se Ruskin aveva creato il mito della Venezia gotica, Codemo predilige la Venezia vista dal basso, rendendo partecipe lo spettatore-lettore di una diversa realtà, quella fabbrile: non più il Canal Grande come crocevia di una élite internazionale ma l'Arsenale con i suoi piroscafi e le moderne e dinamiche compagnie di navigazione.

Negli anni del declino, congeda una «scrittura d'occasione» e la affida al proto affinché la stampi «a mo’ di pennellata, o di romanziere a buon mercato. Pochi centesimi, alla portata di tutti: letteratura alla buona, anche se non è pei letterati». Nelle luci e ombre della cultura di provincia, Codemo, poco interessata alla produzione coeva, non cerca un dialogo con gli scrittori veneti, per esempio Fogazzaro concittadino della madre, ma senza azzardare il mare aperto cerca tutela sottoriva, restando fedele alla sua «letteratura a un soldo» per il popolo. E incalza il lettore fin dentro la sua piccola ma attrezzata officina: «Intanto scrivevo, scrivevo, dipingendo il parco vicino, la dama lontana, mescolando, alterando, traviando, forse ripetendomi, non sapendo cosa facessi il più delle volte, senza perdere per altro mai il filo del racconto e la sua essenza. Verità e sentimento» (Dal parco all'orto, in CODEMO 1892, 3 e 5).

Le sue Notizie al lettore la riconfermano scrittrice addestrata a «l'esigenza della colonna» sia essa la Strenna, il periodico o la misura del romanzo d'appendice, insomma la necessità di «lavorare colla scopa, e non curare che l'effetto», come confessa in una lettera a Caterina Percoto (PERCoto - Codemo 1985, 73).

La corrispondenza epistolare con Caterina Percoto, avviata da una lettera pubblicata contemporaneamente su «La donna e la famiglia» e sul «Giornale delle famiglie - La Ricamatrice» del 16 novembre 1863, rappresenta una perfetta cartina di tornasole per comprendere la differente indole delle due scrittrici e la 
difforme modalità di essere nel mondo. Percoto è modesta, schiva, pudica nello svelare i propri sentimenti, restia a mostrarsi, a tratti quasi reticente, incapace di concedersi a cicalate retoriche come invece è consuetudine di Luigia Codemo, abituata a lasciar andare la penna senza discrezione alcuna. Donna volitiva, caparbia ma a tratti invadente, Codemo sembra presa in un vortice di affermazione di sé che intimidisce Caterina, inducendola a scusare davanti all'amica la sua ritrosia, la sua «umiliante impotenza». La dismisura, la mancanza di discrezione nelle avances di Codemo mostrano per antifrasi tutti i limiti della sua pratica di scrittura, proprio là dove si celebra come «infaticabile operaia». Percoto, per tenere a bada il profluvio di parole dell'amica, cerca in tutti i modi di schermirsi, accusa la sua inettitudine alle alte disquisizioni letterarie, si scusa per i ritardi nelle risposte e per la difficoltà a soddisfare l'insistente richiesta di un «ritratto», un vezzo aristocratico per lei costretta ad occuparsi di contingenze serie per la sopravvivenza propria e dei nipoti. Lamenta la sua malferma salute («la penna mi pesa») e cerca di placare l'interlocutrice con un timido: «invidio la sua operosità, io invece sono un'infingarda solenne e studio e scrivo ben poco» (Рercoto - CODEmo 1985, 65).

Il ritratto morale di Luigia Codemo è quello di un'attivista animata dalle migliori intenzioni; una donna sorprendente nella ostentazione intransigente del suo desiderio di voler «far del bene» ad ogni costo, zelante nell'occuparsi degli affari altrui sempre parlando "a fin di bene», insomma una donna Prassede approdata in laguna. Possedeva una forza e una determinazione speciale nel difendere la famiglia e la tradizione della donna oblativa, sottomessa all'autorità maritale, la donna «con gli occhi bassi e il capo chino», che secondo un proverbio veneto doveva essere bella ma soprattutto «saper tacere e starsene in casa». Un'«acerba predicatrice», per dirla con Manzoni, che conosce bene la retorica di «operare sull'animo» secondo i principi della morale cattolica ed è persuasa che «agli uomini [e alle donne - aggiungiamo pure] il bene bisogna, le più volte, farlo per forza».

Luigia Codemo fatica a cogliere una visione stereoscopica, tridimensionale della condizione femminile del suo tempo. Vede nella donna il determinismo della funzione biologica che la vincola alla funzione di moglie e madre; tuttavia, seguendo le orme materne e le proposte educative suggerite nella Treviso degli anni '6o dagli scritti di Malvina Frank sulle colonne dell'«Archivio domestico», 
conviene sulla necessità di perfezionare e supportare l'istruzione femminile perché è la donna l'anello della catena che consente di incidere sulle nuove generazioni.

Negli anni in cui Anna Maria Mozzoni alza intrepida la voce facendosi avvocata di se stessa e di tutte le proprie simili e, rivolta alle donne abbienti e colte, afferma convinta non potersi definire lavoro «la insignificante direzione di una casa o le industrie di Aracne», Codemo che pure intrattiene una corrispondenza con Dora d'Istria, donna coltissima e pensatrice originale, che in quegli anni aveva dato alle stampe a Parigi, ricevendone una lusinghiera accoglienza, la sua opera Des femmes par une femme (Paris, Librairie Internationale, 1865), si tiene arroccata su posizioni abbastanza conservatrici.

Un capitolo quasi sconosciuto della sua biografia è quello inerente l'attività filantropica, grazie alla quale ha potuto intersecare un protagonismo femminile nuovo nella Venezia degli ultimi decenni dell'Ottocento. Donne aperte al nuovo, capaci di trasformare l'eredità mazziniana in una dimensione etica inedita che si viene via via specializzando in ambito pedagogico educativo, in particolare con le iniziative di Rosa Piazza e Laura Goretti Veruda per la formazione di buone madri e l'istituzione di scuole professionali femminili. Pur senza confondersi con il gruppo veneziano delle «emancipatrici», Codemo già nel marzo 1871 prende posizione sul tema dell'emancipazione e dell'educazione della donna con una lettera aperta pubblicata sulle colonne dell' «Archivio Domestico» di Treviso (IV, 1871, 29, pp. 229-32). Ad offrirle il pretesto è un pamphlet di Aurelia Folliero De Luna, una delle firme più autorevoli dell'emancipazionismo fiorentino, erede delle idee di Luisa Amalia Paladini ma su posizioni più radicali. La Folliero De Luna, che di lì a pochi mesi avrebbe dato vita ad una rivista intitolandola alla madre dei Gracchi, «Cornelia» (sottotitolo: «Rivista letteraria educativa dedicata principalmente agli interessi morali e materiali delle donne italiane»), poneva al centro della sua riflessione la figura della «madre educatrice» con un pressante appello alle donne, ribadito poi nell'editoriale del primo numero della rivista, ad uscire di minorità e a prendersi in mano il proprio destino istruendosi e preparandosi ad istruire, educandosi e nel contempo preparandosi ad educare per costruire un futuro migliore alle donne italiane. 
In questa circostanza la scrittrice trevigiana espone, per la prima volta, le sue convinzioni sull'emancipazione della donna. Si dichiara favorevole alle professioni per le donne in grado di sottrarre le fanciulle all'inoperosa clausura dei conventi, restituendole alla società civile dove possono adempiere ad un'azione intelligente, utile a sé, alla famiglia, alla società. Anzi dichiara in diverse occorrenze essere preferibile che le donne vengano tolte alla famiglia con le professioni piuttosto che con la coercizione dei conventi. Ma una volta ribadita la centralità della famiglia fondata su un matrimonio ben assortito, chiama a testimone George Sand per sancire l'autorità maritale e l'indissolubilità del matrimonio, prendendo decisamente le distanze da quegli «emancipatori» che - a suo avviso - incrinando l'autorità maritale o propugnando il divorzio vorrebbero alterare l'ordine sociale. Pur condividendo la necessità dell'esercizio delle professioni per le donne, Codemo si situa su una lunghezza d'onda lontana dalla tensione progressista e radicale di Aurelia Folliero De Luna. Per quest'ultima si tratta di un «diritto» che emancipa la donna facendole trovare il giusto spazio nella società civile; per la scrittrice trevigiana invece è un «dovere voluto dalle esigenze d'una civiltà progredita». Codemo sembra aver assimilato alcune idee femministe già espresse dalla sua concittadina Malvina Frank (FrANK 1869) in un volume dove propone una «ginnastica intellettuale e morale» alle giovani donne come forma di disciplinamento della ragione e della volontà e di addestramento pratico alla conduzione di una saggia economia domestica (CHEMELLO 1995). Di fatto, recepisce solo una parte della proposta emancipatrice e progressista della Frank. Codemo applica l'etica del lavoro esclusivamente alla donna operaia, secondando la regola aurea del selfelpismo cattolico, «aiutati che Dio t'aiuta», che vede nel lavoro la leva di Archimede capace di emancipare il povero (il «pitocco») dalla miseria, sottraendolo all'obbrobriosa consuetudine di stendere la mano per elemosinare il pane quotidiano.

Nel suo orizzonte di senso non esclude l'esistenza di «donne straordinarie» o di «eroine» purché «la eccezione non diventi regola, e l'ingegno non iscusi la sregolatezza». A conclusione della lunga epistola, Luigia Codemo riconduce la donna dentro il tiepido e protettivo recinto delle pareti domestiche, dove, non più schiava, rimane al riparo dalla sregolatezza. Su tutto vigila l'ombrello protettivo della precettistica cattolica che prende le distanze dalla «Babele protestante», molto lontana dal sentire laico e progressista di Caterina Percoto. 
Nel suo sistema narrativo la Bildung intesa come percorso formativo, come itinerario di maturazione personale, non viene mai declinata al femminile. Difficile leggere i suoi romanzi come «racconti del divenire», nella accezione bachtiniana, per le donne. Il moralismo di Codemo non ammette deroghe. La trasgressione femminile, secondo i migliori stereotipi della letteratura d'appendice, non può che condurre alla pazzia, epilogo e punizione insieme per il disordine provocato dal proprio comportamento (vd. Fiore di serra). La padrona di casa non può «starsene tre ore allo specchio», deve assolvere le incombenze domestiche, deve restare nei ranghi senza atteggiarsi a «gran dama» o osare varcare la soglia della pudica decenza (CODEMO 186ob, 39-40).

Qualche considerazione a margine deve farsi sul tema dell'amore coniugale, di frequente descritto nelle sue nascoste contraddizioni e nelle sue crepe profonde come una burrascosa esperienza. Al centro di queste relazioni abitate da contraddizioni insanabili, piuttosto che da un amore quietamente costante e fedele, stanno altrettante figure di donna. Ma Codemo si mantiene lontana dall'indagine introspettiva, dall'effusiva confessione di sé. Anche quando si cimenta in bilanci coniugali à rebours, le intermittenze del cuore femminile sono poco compenetrate dalla sua penna. Il suo calamaio rimane ostaggio di remore e pregiudizi di una religiosità un po' bigotta che raffrena ogni libero esercizio creativo. E le citazioni, anche le più trasparenti, di modelli letterari di alto profilo risultano povere di espansività sentimentale.

Il personaggio di Fiorenza, nel romanzo La rivoluzione in casa (CODEMo 1869), è forse la figura più riuscita, quella capace di esprimere una mediazione possibile tra un romantico amor di patria e una sofferenza inaudita del cuore per la separazione dall'amato, ma finisce col mostrarsi candidamente indifesa oltre la soglia del «piccolo, ma caldo paese, che, per esse [scil.: le donne] costituiva il mondo». Fiorenza, giovane sposa e madre emblema dell'oblatività femminile, separata suo malgrado dal marito impegnato ad inseguire l'ideale risorgimentale, è:

umile creatura di Dio, [...] non poteva che abbassar gli occhi davanti allo splendore d'una stella così viva, e fare atto di assoluta docilità, in faccia ad un ineluttabile decreto. Ella sedeva solitaria sulla soglia della casa, per rimanerci, appunto secondo le antiche istituzioni italiane, genio tutelare di essa. (CODEMO $1869,190)$ 
Accanto a questa figura, due sono i personaggi femminili che, nella sequenza delle Scene e descrizioni, possono inscriversi in una esemplare tipologia di donne. La prima è Marcella, ne I miracoli del sentimento (CODEMO 1871), una aristocratica colta e raffinata, donna di una profonda spiritualità e di lucida e calda umanità, capace di costruire relazioni profonde e consonanze intellettuali con le proprie simili fino a farsi testimonianza vivente di amore e di fede. L'altra è la Checca, nel racconto lungo Anzù, espressione della tipica cordialità veneta, una donna di umili origini, sensuale e incolta, istintiva ma dotata di senso pratico; una donna determinata a far del bene a tutti i costi magari con un po' di invadenza, una riuscita "figurina" alla Faldella, ma declinata al femminile.

Nella sua tensione a comporre e a far stampare «un libro da leggere», un libro da far collocare «nella sporta della spesa», Codemo pur avendo letto e riletto a lungo negli anni della sua formazione Dante, George Sand, Balzac e Manzoni, assieme alle pagine di Giusti e alla Povera tosa di Carcano, non esplora lo spazio reale e simbolico della donna che sta movendo i primi passi per affermare il proprio diritto alla parola e ad un'esistenza non contristata. La figurina che orna il frontespizio delle «scene domestiche» intitolate Nuovi ricchi è una fanciulla con un libro aperto tra le mani in atto di leggere, ma nelle «scene della vita» descritte e rappresentate nelle ponderose pagine di Luigia Codemo la «donna di studio», la lettrice appassionata e desiderosa di apprendere è confinata nel limbo della mera eccezione.

\section{Bibliografia citata:}

ARGONDIZZA1898:

Antonio ArgondizZA, Necrologio, in «Il Popolano» di Corigliano Calabro, 28 agosto 1898, p. 3.

CACCIANIGA 1875:

Antonio CaCcIaniga, Reminiscenze dall'esilio, Milano, Treves, 1875, pp. 164-68.

CASTELnUOVO 1872:

Enrico Castelnuovo, Prefazione a Racconti e bozzetti, Firenze, Le Monnier, 1872.

CHEMELlO 1995:

Adriana CHEMELLO, «Libri di lettura» per le donne. L'etica del lavoro nella letteratura di fine Ottocento, Alessandria, Edizioni dell'Orso, 1995. 
CODEMO 1856:

Luigia Codemo, Le memorie d'un contadino. Scene domestiche, Venezia, Nel priv. Stabil. di G. Antonelli, 1856, 2 tomi [ $2^{\text {a }}$ ed.: Venezia, Cecchini, 1874

CODEMO 1858:

Luigia Codemo, Berta / Prima cronaca d'una anonimo. Scene domestiche, Venezia, Naratovich, 1858 [Altra ed.: Venezia, Stab. Tip. Lit. di Colombo Coen Ed., 1874].

CODEMO 1860a:

Luigia CODEMo, Fiore di prato. Seconda cronaca d'un anonimo. Scene domestiche, Venezia, Tip. del Commercio, 1860 [Altra ed.: Venezia, Stabilimento Tip. Lit. di C. Coen Editore, 1875].

CODEMO 186ob:

Luigia CODEMO, Fiore di serra. Terza cronaca d'un anonimo. Scene domestiche, Venezia, Tip. del Commercio, 1860 [Altra ed.: Venezia, Stabilimento Tip. Lit. di C. Coen Editore, 1875].

CODEMO 1865:

Luigia CoDemo, Miserie e splendori della povera gente Scene popolari del Veneto, Rovereto, Caumo, 1865 [ $3^{\text {a }}$ ed.: Venezia-Trieste, Stabilimento Libr. Tip. Lit. di C. Coen Editore, 1876].

CODEMO 1867:

Luigia Codemo, I Miracoli del sentimento. Scene del primo sacco di Roma, Treviso, Tip. Giovani Abbandonati, 1867.

CODEMO 1869:

Luigia CoDEmo, La rivoluzione in casa Scene di vita italiana, Venezia, Cecchini, 1869 [2 $2^{\text {a }}$ ed.: ivi, 1872; $3^{\text {a }}$ ed.: Treviso Zoppelli, 1888; ed. postuma, a c. di Raffaello Barbiera, Milano, Treves, 1906.

CODEMO 1870:

Luigia CODEMO, Dal mare alle Alpi, illustrazione d'un album, in «Strenna Veneziana per l'anno 1870», Venezia, Tip. del Commercio, 1869, 141-75.

CODEMO 1871:

Luigia Codemo, Scene e descrizioni illustrate, Venezia, Tipografia del Commercio, 1871.

CODEMO 1872:

Luigia CODEMO, Fronde e Fiori del Veneto Letterario in questo secolo. Racconti biografici, Venezia, Tipografia di Giuseppe Cecchini e C., 1872.

CODEMO 1875:

Luigia CoDEMo, Pagine Famigliari Artistiche Cittadine (1750-1850), Venezia, Tipografia del Commercio di M. Visentini, 1875 [Altra ed., con integrazioni e aggiunte: Treviso, coi tipi di Luigi Zoppelli, 1878].

CODEMO 1884:

Luigia CoDemo, Nohant. Tocchi pittoreschi, Treviso, Tipografia Luigi Zoppelli, 1884 .

CODEMO 1891:

Luigia CoDEMo, Venticinque anni dal 19 ottobre 1866, Venezia, Tip. Visentini, 1891.

CODEMO 1892:

Luigia Codemo, Dal parco all'orto. Scene intime, Venezia, coi tipi dei fratelli Visentini, 1892.

CODEMO 1895:

Luigia CoDEMO, Pennellate marinare, Venezia, Visentini, 1895.

CODEMO 1996:

Luigia Codemo, Scene di vita. Antologia di narrativa, a cura di G. Pullini, VeneziaMirano, Eidos, 1996. 
CROCE 1924:

Benedetto CROCE, Conversazioni critiche. Serie seconda, Bari, Laterza, $1924^{2}$.

DE GUBERNATIS 1875:

Angelo DE GUBERNATIS, Dizionario biografico degli scrittori contemporanei, Firenze, Le Monnier, 1879, pp. 300-01.

FRANK 1869:

Malvina FrANk, Le Fidanzate. Saggio sulla educazione della donna, Treviso, Edizioni dell'Archivio Domestico di Treviso, 1869.

NiEvo 1956:

Ippolito NIEvo, La nostra famiglia di campagna, in Novelliere campagnuolo e altri racconti, a c. di Iginio De Luca, Torino, Giulio Einaudi Editore, 1956.

MOLMENTI 1882:

Pompeo G. Molmenti, Vecchie storie, con disegni di Giacomo Favretto, Venezia, Ferd. Ongania, Editore, 1882, 149-60.

MUSATTI 1898:

Cesare MUSATTI, Luigia Codemo. Profilo, in «Ateneo Veneto», XXI, 2 (1898), 24560.

PAGANO 1991:

Esmeralda PAgano, Ottocento al femminile. Lettere inedite di Luigia Codemo a Giannina Milli, in «Critica letteraria», XIX, 72 (1991), 567-88.

PERCOTO - CODEMO 1985:

Le umili operaie. Lettere di Luigia Codemo e Caterina Percoto, a c. di Rossana Caira Lumetti, Napoli, Loffredo, 1985.

SAND 1846:

George SAND, La mare au diable, Bruxelles, Meline, Can set compagnie, 1846.

TOMMASEO 1867:

Niccolò TOMmASEO, Una lettera, in «Il lavoro», VIII, 1867, p. 337.

Note

${ }^{1}$ Luigia Codemo nasce il 5 settembre 1828 a Treviso da Michelangelo Codemo, uomo di lettere e discreto narratore, e Cornelia Sale, vedova Mocenigo, cultrice delle lingue classiche, traduttrice e poetessa. Può vantare, da parte di madre, una ascendenza illustre in quanto nipote di Fiorenza Vendramin Sale, la «bella suicida» il cui ritratto rimane ibernato nelle pagine di Molmenti, assieme ad altre Vecchie storie (MOLMENTI, 1882, 149-60). Nelle sue Pagine famigliari, avvertendo il lettore di non aver voluto scrivere «un'opera letteraria» ma un «buon ricordo», Luigia dedica la prima parte, intitolata Pagine intime, ad una ricostruzione genealogica delle due famiglie paterna e materna, ma mentre esaurisce con brevi note la storia pregressa della prima, discendente dalla piccola nobiltà trentina insediatasi nel corso del XVII secolo a Pederobba, molto più spazio occupa la narrazione dell'avventuroso «romanzo» della sua ava, Fiorenza Vendramin, verso la quale sente un affettuoso e malinconico attaccamento. Nel riandare alle di lei care memorie, la nipote la paragona a «la Gaspara Stampa, la Saffo della Laguna», e sfoglia con commosso rimpianto le poche carte a lei sopravvissute: cinque egloghe pastorali, un dramma poi musicato dal cognato, il marchese Ricci, alcune pagine di confessioni in francese, una cantata e poesie varie, oltre ad un ritratto, anch'esso in lingua francese, steso dalla sorella Maria Vendramin (CODEMO 1875).

${ }^{2}$ La presenza magistrale di entrambi i genitori è fondamentale nella formazione letteraria di Luigia, che ricorda: «a questo proposito mi piace dire quanto divergenti le opinioni dei miei genitori, perché mia madre mi porse l'Eneide, il papà i Promessi sposi: 
essi credevano darmi gli antipodi e mi davan babbo e figliuolo, o meglio nonno e nipote. I Promessi sposi divennero il mio pane quotidiano, dacché li lessi la prima volta, li rileggo ogni anno, li so a memoria, e sempre vi scopro qualcosa di nuovo, e ogni volta benedico l'ispirazione di quel divino, che li ha composti» (CODEMo 1875, 263). Dalle Pagine famigliari apprendiamo ancora che a undici anni aveva imparato a memoria circa quattrocento versi dell'Eneide con relativa traduzione e una decina di canti della Commedia dantesca. Un ambiente familiare vivace e stimolante quello di Luigia, dove alle letture si intrecciano le amicizie e le conversazioni con poeti e letterati di area veneta.

3 Manca soprattutto una puntuale ricognizione degli scritti vari dispersi in riviste, giornali a cui Codemo ha collaborato seppur con discontinuità, così come dei contributi apparsi in strenne o in opuscoli d'occasione, di cui conosciamo solo la punta di un iceberg ancora sommerso. Alcune informazioni di prima mano sulla storia editoriale dei suoi lavori si ricavano dalla lettura attenta delle sue Pagine famigliari (CODEMO 1875). 4 Il romanzo viene segnalato da una recensione di Pietro Selvatico per la «Nuova Antologia», a. V, vol. XIV, 5 (1870), 153-56; ne parlano favorevolmente Niccolò Tommaseo (Una lettera, in «Il lavoro», VIII, 1867, p. 337), Angelo De Gubernatis (Dizionario biografico degli scrittori contemporanei, Firenze, Le Monnier, 1879, pp. 300-01), Enrico Castelnuovo (nella Prefazione a Racconti e bozzetti, Firenze, Le Monnier, 1872), Antonio Caccianiga (in Reminiscenze dall'esilio, Milano, Treves, 1875, pp. 164-68) che ricorda con nostalgia, nelle sue pagine autobiografiche, le conversazioni serali in casa Codemo, durante il soggiorno della famiglia di Luigia sulle rive della Senna nell'autunno inverno del 1849, e Dora d'Istria (pseudonimo della principessa valacca Elena Ghika). 